\title{
Uses of History of Mathematics in School (Pupils Aged 6-13)
}

\author{
Bjørn Smestad
}

\section{Report}

Activities concerning history of mathematics have been a part of ICMEs since ICME2 in Exeter in 1972. They are now regular features of ICMEs, organized by the HPM (International Study Group on Relations between History and Pedagogy of Mathematics). The premise of this discussion group was that research on history of mathematics in education tends to have older pupils and students in mind, and that there is a lack of both research and resources on how to include a historical perspective when teaching younger pupils.

Three key questions were pointed out in the invitation to the discussion group:

- Which ideas from HPM can be used with children (aged 6-13) in such a way that produces good results (e.g. improved student engagement, positively impacted student learning)?

- What would be criteria for finding, developing and selecting materials to be used with children (aged 6-13)?

- How does the HPM community in particular (and mathematics education community more broadly) assure that high-quality material that cover a variety of topic are produced and shared?

Question 1 was discussed in the first session and questions 2 and 3 were discussed in the second session.

Organizers Co-chairs: Bjørn Smestad (Norway), Funda Gonulates (USA); Team Members: Narges Assarzadegan (Iran), Kathy Clark (USA), Konstantinos Nikolantonakis (Greece); Liaison IPC Member: Evelyne Barbin (France).

\section{B. Smestad ( $\square)$}

Oslo and Akershus University College of Applied Sciences, Oslo, Norway

e-mail: bjorsme@hioa.no 
In the first session, Kathy Clark gave a short introduction of ideas from the vast literature on how and why to include history of mathematics in teaching. Thereafter, Narges Assarzadegan gave a short talk on how she has been working with her students in Iran on the topic. Kathy Clark subdivided question 1 into further subquestions: What are the ideas for which HPM contributes meaningfully to the mathematical experience of pupils aged 6-13? What are the forms of good results we wish to happen? How do we know when good results occur? What are some of the obstacles that teachers using HPM with pupils of this age may encounter-and what are ways to address or minimize the obstacles?

Group discussions on the first question brought forward a wealth of ideas: the use of historical instruments, finding good problems from history to engage children of this age range, using concrete materials to visualize mathematics, working with words instead of symbols, exploring cross-curricular themes (for instance historical measuring units), using source material from the middle ages, studying materials from the cultures of children's parents and grandparents, and studying positive/negative numbers through history, to mention a few. More generally, it was pointed out that although "storytelling" was in our introduction described as just one of many ways of working with history of mathematics to kids, storytelling is indeed particularly important at this age level and should not be disparaged. Teachers that are able to fascinate their pupils with great (and meaningful) stories from the history of mathematics have a wonderful gift.

The good results participants wish to happen at this age level mostly has to do with the attitudes of the children: we want them to see mathematics as a fascinating cultural and human activity and make them connect to it in new ways. We will probably never be able to prove beyond doubt that using history of mathematics with children do have positive effects, as history of mathematics will always be just one of several elements a teacher uses simultaneously to engage his students. For the teacher, however, such proofs are not necessary-just seeing the pupils engaged is good enough.

Of course, there are obstacles - both in terms of resources and in teachers' opinion that history of mathematics will take time from mathematics. Moreover, as work on history of mathematics is not mandated in curricula in most countries, there is the ever-present need to justify it to colleagues who are not interested. This can also be lonely work. Some of these issues can partly be remedied by working on what we discussed in session 2 , however.

In the second session, as an introduction to discussions on question 2 and 3, Bjørn Smestad and Kathy Clark gave some good examples of use of history of mathematics in teaching, including some from online sources.

The group came up with a long list of criteria for materials, noting that not every resource need to fit every criterion. The resource should:

- Include significant mathematics (and be curriculum-related)

- Include activity/task/problem/something for pupils to "do"

- Fire-up the imagination; inspire pupils to do mathematics

- Tell a story 
- Have multiple representations (pictures, text, sound, video, interactivity)

- Show mathematics as a human endeavor (e.g., have a cultural aspect)

- Be doable in a "reasonable amount of time"

- Generate discussion, debate among the pupils

- Be authoritative and accurate

There are lots of materials on the internet, and at first you feel lost as it is difficult to see what is of good quality. After a while, you start being able to determine what "makes sense", but still you need to sort through a lot of bad stuff while looking for the gems. (But to get even there, you will probably need experience in using the materials - and where do you get that?) Thus, there is a need of a "clearing house" for keeping valuable materials in one location. This idea was developed further later in the discussion: what we need is a "Kantor project" (named after Moritz Kantor), mimicking the "Klein Project" in providing high-quality resources to teachers, for instance with comments both from historians of mathematics and from teachers who have used the resources with pupils (including information on how it was used and the perceived outcomes). In addition, the need for History of Mathematics courses and better resources at libraries, were mentioned.

The discussion group consisted of about 25 people from around the world, with a good mix of well-known faces in the HPM community and newcomers. This lead to good discussions where everybody took part. In that respect, we view the discussion group as a successful experience, and hope that the discussions here will inspire further work on teaching with history of mathematics for young pupils. We do hope there will be increased dissemination of ideas for this purpose in the years to come.

This report was written by Bjørn Smestad. He is happy to be contacted at bjorsme@ hioa.no for further information on the works of this DG.

Open Access This chapter is distributed under the terms of the Creative Commons Attribution Noncommercial License, which permits any noncommercial use, distribution, and reproduction in any medium, provided the original author(s) and source are credited. 